\title{
MicroRNA-139-5P inhibits human prostate cancer cell proliferation by targeting Notch1
}

\author{
QIAN SUN* ${ }^{*}, D_{A N H U I ~ W E N G}{ }^{*}$, KEZHEN LI, SHUANG LI, XIANGYANG BAI, \\ CAN FANG, DANFENG LUO, PENG WU, GANG CHEN and JUNCHENG WEI \\ Cancer Biology Research Center, Tongji Hospital, Tongji Medical College, \\ Huazhong University of Science and Technology, Wuhan, Hubei 430030, P.R. China
}

Received December 9, 2015; Accepted April 11, 2018

DOI: $10.3892 / \mathrm{ol} .2018 .8773$

\begin{abstract}
Despite an improvement in the efficacy of chemotherapeutic agents, the outcome of patients with prostate cancer remains poor. MicroRNA (miRNA/miR)-139 expression is often downregulated in multiple types of tumor, including in prostate cancer. The aim of the present study was to investigate the inhibitory effect of miR-139 on the PC-3, C4-2B and LNCaP prostate cancer cell lines. Analysis of the cell cycle of PC-3, C4-2B and LNCaP cells transfected with miR-139 revealed a significantly increased percentage of cells in the $\mathrm{G}_{1}$ phase and a decreased percentage in the $\mathrm{S}$ and $\mathrm{G}_{2}$ phases compared with those transfected with a negative control miRNA. The growth inhibitory rate of miR-139-transfected cells 24,48 and $72 \mathrm{~h}$ after transfection were $32.83 \pm 2.61$, $52.58 \pm 3.2$ and $62.36 \pm 4.55 \%$ in PC-3 cells; $30.28 \pm 2.25$, $51.74 \pm 3.27$ and $60.80 \pm 3.58 \%$ in $\mathrm{C} 4-2 \mathrm{~B}$ cells; and $33.20 \pm 2.67$, $51.83 \pm 3.59$ and $61.79 \pm 4.85 \%$ in LNCaP cells, respectively. The present study revealed that miR-139 inhibited the proliferation of prostate cancer cells by interfering with the cell cycle. Further study into the mechanism by which this happened suggested that miR-139 reduced cyclin D1 expression and inhibited cell proliferation through targeting Notch1.
\end{abstract}

\section{Introduction}

Prostate cancer (PCa) remains the most commonly diagnosed cancer and the third most common cause of cancer-associated mortality among males in western countries (1). In China, the incidence rate of PCa is increasing year by year and has already exceeded the incidence of bladder cancer (2). The

Correspondence to: Dr Juncheng Wei, Cancer Biology Research Center, Tongji Hospital, Tongji Medical College, Huazhong University of Science and Technology, 1095 Jiefang Avenue, Wuhan, Hubei 430030, P.R. China

E-mail:wjcwjc999@126.com

*Contributed equally

Key words: microRNA-139, proliferation, Notch1 majority of patients with middle/late stage or recurrence of PCa exhibit androgen-independent growth and resistance to multiple therapies, which leads to the failure of conventional treatment and a high mortality rate (3). Therefore, there is an urgent requirement to identify effective biomarkers to strengthen the efficiency of early diagnosis and improve the therapeutic strategies used to treat $\mathrm{PCa}$ (4).

MicroRNAs (miRNAs/miRs) are endogenously expressed, small non-coding RNAs that regulate target gene expression, cell proliferation, cell differentiation and cell apoptosis through combination with the 3'-untranslated region (3'-UTR) of target mRNAs to induce mRNA degradation. Previous studies have revealed an association between the expression of various miRNAs and tumorigenesis, invasion, metastasis, resistance to chemotherapy and poor prognosis $(5,6)$.

There is evidence linking the reduced expression of miR-139 to cancer progression and carcinogenesis (7). For instance, miR-139 inhibits cell proliferation and invasion by targeting the expression of insulin-like growth factor 1 receptor in non-small cell lung cancer (8). miR-139 also exerts a tumor suppressor function by targeting Notch1 in colorectal and breast cancer $(9,10)$. To the best of our knowledge, the effects of miR-139 in PCa, and the molecular mechanisms underlying these effects, remain elusive. Therefore, the present study aimed to investigate the biological functions of miR-139 on the cell cycle, proliferation, apoptosis, migration and invasion in $\mathrm{PCa}$.

\section{Materials and methods}

Cell lines and tissue samples. The $\mathrm{PCa} \mathrm{PC}-3, \mathrm{C} 4-2 \mathrm{~B}$ and $\mathrm{LNCaP}$ cell lines were grown in a $37^{\circ} \mathrm{C}, 5 \%(\mathrm{v} / \mathrm{v}) \mathrm{CO}_{2}$ growth chamber. Cells were cultured in Dulbecco's modified Eagle's medium (DMEM) supplemented with $10 \%$ fetal bovine serum (FBS), $100 \mathrm{U} / \mathrm{ml}$ penicillin, $100 \mu \mathrm{g} / \mathrm{ml}$ streptomycin solution. All cell culturing reagents were obtained from Invitrogen; Thermo Fisher Scientific, Inc. (Waltham, MA, USA).

Tissue samples [10 samples of benign prostatic hyperplasia (BPH) and 10 samples of $\mathrm{PCa}$ ] were obtained from patients who underwent surgical treatment by transurethral resection of the prostate or radical prostatectomy between January 2012 and October 2013. The age of the patients was $65.6 \pm 3.5$ years. The tissue samples were collected by the Department of 
Pathology, Tongji Hospital, Tongji Medical College, Huazhong University of Science and Technology (Wuhan, China). The histopathological diagnosis was reviewed by at least two specialists in pathology. Tissue samples were obtained and handled in accordance with a protocol approved by the Institutional Review Board for Human Research of Tongji Hospital (Wuhan, China). Written informed consent was obtained from all patients.

Reverse transcription-quantitative polymerase chain reaction. miRNAs were isolated using the miRNeasy Mini kit (Qiagen Inc.) according to the manufacturer's protocol. miRNAs were assayed using TaqMan MicroRNA assays (Thermo Fisher Scientific, Inc.) in accordance with the manufacturer's protocol. Samples were normalized to U6 small nuclear RNA (Applied Biosystems; Thermo Fisher Scientific, Inc.). The comparative $\mathrm{Cq}$ method (11) was used to calculate the relative changes in gene expression on the Applied Biosystems ${ }^{\circledR} 7500$ Fast Real Time PCR system (Applied Biosystems; Thermo Fisher Scientific, Inc.) using the following thermocycler program for all genes: $5 \mathrm{~min}$ of pre-incubation at $95^{\circ} \mathrm{C}$ followed by 40 cycles of $15 \mathrm{sec}$ at $95^{\circ} \mathrm{C}, 15 \mathrm{sec}$ at $60^{\circ} \mathrm{C}$, and $30 \mathrm{sec}$ at $72^{\circ} \mathrm{C}$. The primers used were as follows: miR-139-5p, forward, 5'-CCTCTACAGTGCACGTGTCTC-3', and reverse, 5'-CGC TGTTCTCATCTGTCTCGC-3'; and U6, forward, 5'-TGC TCGCTTCGGCAGC-3', and reverse, 5'-AAAAATATGGAA CGCTTCACG-3'.

miRNA transfection. Cells were plated in DMEM without antibiotics $\sim 24 \mathrm{~h}$ prior to transfection. Transient transfection of a human miR-139-5p precursor (has-miR-139-5P mimic; cat no. MIMAT0000250; Ambion; Thermo Fisher Scientific, Inc.) or miRNA mimic negative control (miR-CN; cat no. AM7110; Ambion, Thermo Fisher Scientific, Inc.) was performed using Lipofectamine 2000 (Invitrogen; Thermo Fisher Scientific, Inc.) according to the manufacturer's protocol.

Apoptosis assays. Apoptosis was assessed by measuring the membrane redistribution of phosphatidylserine using an Annexin V-FITC Apoptosis Detection kit (BD Pharmingen; BD Biosciences, San Jose, CA, USA). A total of $1 \times 10^{6}$ cells were collected, washed twice with PBS and resuspended in $500 \mu \mathrm{l}$ of the kit's staining solution, containing fluorescein isothiocyanate-conjugated Annexin V antibodies and propidium iodide (PI). Following incubation on ice for $30 \mathrm{~min}$, the cells were analyzed using a BD FACSCalibur flow cytometer and CellQuest Pro software (version 5.1; BD Biosciences). Basal apoptosis and necrosis rates were determined in untranfected cells.

Cell cycle analysis by fluorescence activated cell sorting. Cells were trypsinized, washed twice in ice-cold PBS, and fixed with cold $70 \%$ ethanol for $24 \mathrm{~h}$. Propidium iodide solution $(40 \mu \mathrm{g} / \mathrm{ml})$ and RNase A (cat. no. EN0531; Thermo Fisher Scientific, Inc.) were added to the cells, which were then incubated for $45 \mathrm{~min}$ in the dark at $4^{\circ} \mathrm{C}$ prior to analysis.

Cell proliferation assay. Cells were seeded at a density of $2 \times 10^{3}$ per well in 96-well plates and incubated in DMEM containing $10 \%$ FBS. Following seeding, cells were transfected as aforementioned. The tetrazolium salt MTT was added to the wells, cells were incubated for $4 \mathrm{~h}$ at $37^{\circ} \mathrm{C}$, then the medium was changed with $150 \mu \mathrm{l}$ dimethyl sulfoxide at room temperature to dissolve the formazan crystals, the optical density (OD) was measured at $490 \mathrm{~nm}$ using a microplate reader. The growth inhibitory rate $=(1-\mathrm{OD}$ value of the observed group $/ \mathrm{OD}$ value of the control group) $\times 100 \%$.

Wound healing assay. Cells were grown on 6-well plates. At $24 \mathrm{~h}$ after transfection, the confluent cells were carefully scratched using a $200 \mu \mathrm{l}$ pipette tip. Images were captured in 8 different regions per well at 0 and $48 \mathrm{~h}$ using a Zeiss Axiovert 200 M (Zeiss AG, Oberkochen, Germany) inverted light microscope. For each region, the distance migrated by the cells was measured at 3 different points. The migrated distance was recorded as a mean of 24 points every hour. Then, the formula [mean speed $(\mu \mathrm{m} / \mathrm{min})=$ migration distance/migration time] was used to assess the migration speed of tumor cells.

Cell invasion assay. The cell invasion assay was performed using Boyden Transwell chambers ( $8.5 \mathrm{~mm}$ in diameter) with Matrigel added to the filters (pore size, $8 \mu \mathrm{m}$; Costar; Corning Incorporated, Corning, NY, USA). Briefly, $500 \mu 1$ DMEM containing $10 \%$ FBS was added to the bottom well. At $24 \mathrm{~h}$ after transfection, cells were re-suspended in DMEM without FBS at a concentration of $1 \times 10^{6} \mathrm{cells} / \mathrm{ml}$, and $500 \mu 1$ cell suspension was added to the top well. Following incubation at $37^{\circ} \mathrm{C}$ for $36 \mathrm{~h}$, the cells that had not migrated were removed from the upper surface of the filters using cotton swabs, and those that had invaded to the lower surface of the filters were fixed in $100 \%$ methanol at room temperature for $15 \mathrm{~min}$, followed by staining with $0.05 \%$ crystal violet at room temperature for $2 \mathrm{~h}$. Invasiveness was determined by counting the mean number of cells within five random fields of view using an inverted light microscope (magnification, $\mathrm{x} 100$ ).

Dual luciferase reporter assay. Potential miR-139-5p binding sites were predicted using TargetScan (www.targetscan.org). The sequence of $42 \mathrm{bp}$ segments with the wild-type or mutant 3'UTR seed region of Notch1 (prepared by the deletion of 10 nucleotides in the seed region) was synthesized and cloned into a pMIR-REPORT luciferase vector (Applied Biosystems; Thermo Fisher Scientific, Inc.). The synthesized oligonucleotides were as follows: Wild-type Notch1: 5'-CTAGTGACT TTAAAAGTGATCTACATGAGGA ACTGTAGATGA TGTGAGCT-3'; 5'-CACATCATCTACAGTTCCTCATGT AGATCACTTTTAAAGTCA-3'; Mutant Notch1: 5'-CTAGTG ACTTTAAAAGTGATCTACATGAGTGATGTGAGCT-3'; 5'-CACATCACTCATGTAGATCACTTTTAAAGTCA-3'. The Notch1 construct or control construct was co-transfected into C4-2B and PC-3 cells along with miR-139 or miR-CN using Lipofectamine 2000 (Invitrogen; Thermo Fisher Scientific, Inc.). Firefly luciferase activity was measured $48 \mathrm{~h}$ following transfection and normalized against Renilla luciferase activity, using the Dual Luciferase Reporter Assay system (Promega Corporation, Madison, WI, USA) according to the manufacturer's protocol.

Western blot analysis. Cells were lysed on ice for $30 \mathrm{~min}$ with a lysis buffer containing $150 \mathrm{mmol} / \mathrm{l} \mathrm{NaCl}, 50 \mathrm{mmol} / \mathrm{l}$ 
A

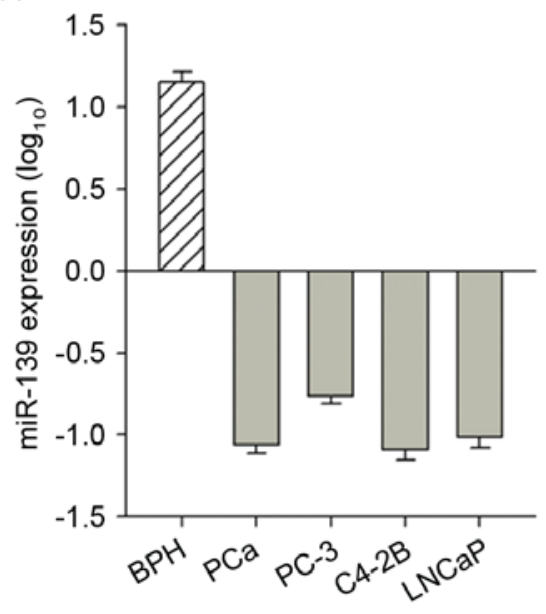

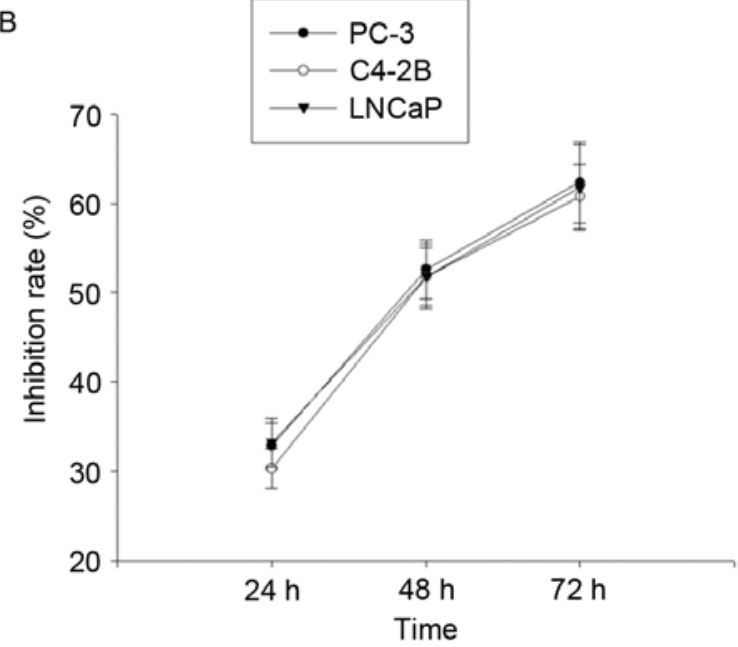

Figure 1. Expression of miR-139-5P in prostatic adenocarcinoma tissues and prostate cancer cell lines. (A) Reverse transcription-quantitative polymerase chain reaction analysis of relative miR-139-5P expression levels in human BPH tissues $(\mathrm{n}=10), \mathrm{PCa}$ tissues $(\mathrm{n}=10)$ and PCa PC-3, C4-2B and LNCaP cell lines. Data were normalized to U6 small nuclear RNA control and represented as the mean \pm standard error of the mean. (B) miR-139-5P inhibited prostate cancer cell proliferation. Cell proliferation rates were detected by MTT assay. miR, microRNA; BPH, benign prostatic hyperplasia; PCa, prostate cancer.

Tris (pH 7.4), 1\% Triton $\mathrm{X}-100,1 \%$ sodium deoxycholate, $0.1 \%$ SDS, and protease inhibitor cocktail (cat. no. S8830; Sigma-Aldrich; Merck KGaA, Darmstadt, Germany), then the product were centrifuged at $14,000 \mathrm{x}$ g at $4^{\circ} \mathrm{C}$ for $30 \mathrm{~min}$. A total of $100 \mu \mathrm{g}$ protein were denatured in SDS sample buffer [2\% SDS, $62.5 \mathrm{mM}$ Tris-base (pH 6.8), 10\% glycerol, $5 \% \beta$-mercaptoethanol, and $0.005 \%$ bromophenol blue] and loaded onto a $10 \%$ SDS-PAGE gel. The separated proteins were transferred onto nitrocellulose membranes and the membranes were blocked overnight at $4^{\circ} \mathrm{C}$ in TBS with $5 \%$ (w/v) powdered skimmed milk, and were stained with the recommended dilution of primary antibodies against Notch1 (cat. no. 3447; dilution. 1:1,000), cyclin D1 (cat. no. 2978; dilution, 1:1,500), MMP7 (cat. no. 3801; dilution, 1:1,000), MMP9 (cat. no. 13667; dilution, 1:1,000) and $\beta$-actin (cat. no. 4970; dilution, 1:1,500) at room temperature for $2 \mathrm{~h}$ (all from Cell Signaling Technology, Inc., Danvers, MA, USA). Following washing, the blots were incubated with a 1:2,000 dilution of goat-anti-rabbit (cat. no. 7074; Cell Signaling Technology Inc.) or goat-anti-mouse (cat. no. 7076; Cell Signaling Technology Inc.) immunoglobulin $\mathrm{G}$ antibodies conjugated to horseradish peroxidase at room temperature for $1 \mathrm{~h}$. The blots were developed with the Enhanced Chemiluminescence Western Blot detection kit (Pierce; Thermo Fisher Scientific).

Transmission electron microscopy. Cell pellets were fixed in $2 \%$ paraformaldehyde and $2.5 \%$ glutaraldehyde in $0.1 \mathrm{M}$ sodium cacodylate buffer $(\mathrm{pH} 7.4)$ at room temperature for $5 \mathrm{~min}$. Firstly, $10 \mathrm{ml}$ 1x PBS was added to a Petri dish containing the cells to briefly wash them. Next, the supernatant was discarded and $1 \mathrm{ml} 1 \mathrm{x}$ fixative was added into the dish at room temperature. Following this, cells were scraped and transferred into a 1.5-ml Eppendorf tube. The cells were then centrifuged at 500-800 $\mathrm{xg}$ at room temperature for $5 \mathrm{~min}$. The pellet was embedded in epoxy resin at $60^{\circ} \mathrm{C}$ for $48 \mathrm{~h}$. Ultra-thin sections $(100 \mathrm{~nm})$ were cut with an ultramicrotome and stained with $5 \%$ uranyl acetate in $50 \%$ ethanol, followed by $2 \%$ aqueous lead citrate at $37^{\circ} \mathrm{C}$ for $30 \mathrm{~min}$. Finally, the ultra-thin sections were observed on a Philips CM12 Microscope (FEI Inc., Eindhoven, Netherlands).

Statistical analysis. Results are reported from at least three different experiments. Statistical analyses were performed with SPSS software version 13.0 (SPSS, Inc., Chicago, IL, USA). For the comparisons between 2 groups (cell cycle assay, cell invasion assay, wound healing assay and dual luciferase reporter assay), Student's t-test was used for analysis. For combination studies (miR-139-5P expression data), one-way analysis of variance followed by least-significant difference post-hoc test was used for analysis. All data are reported as the mean \pm standard error of the mean.

\section{Results}

miR-139 inhibits the proliferative activity of PCa cells. miR-139-5P expression data were detected for $\mathrm{BPH}(\mathrm{n}=10)$ and $\mathrm{PCa}(\mathrm{n}=10)$ patient samples, and the PC-3, C4-2B and LNCaP $\mathrm{PCa}$ cell lines. miR-139-5P expression was higher in $\mathrm{BPH}$ tissue compared with the prostate cancer tissue, suggesting that there was a significant decrease in the expression level of miR-139-5P in PCa ( $\mathrm{P}<0.001$; Fig. 1A). Additionally, the expression of miR-139-5P in the three $\mathrm{PCa}$ cell lines was lower than BPH tissue (Fig. 1A).

The MTT assay results revealed that the growth inhibitory rate [(1-OD value of the observed group/OD value of the control group) $x 100 \%$ ] of miR-139 transfected cells at 24,48 and $72 \mathrm{~h}$ after transfection were $32.83 \pm 2.61,52.58 \pm 3.29$ and $62.36 \pm 4.55 \%$ in PC-3 cells, respectively; $30.28 \pm 2.25$, $51.74 \pm 3.27$ and $60.80 \pm 3.58 \%$ in $\mathrm{C} 4-2 \mathrm{~B}$ cells, respectively; and $33.20 \pm 2.67,51.83 \pm 3.59,61.79 \pm 4.85 \%$ in LNCaP cells (Fig. 1B). The inhibition rate of the three transfected PCa cell lines increased over time, and it was concluded that miR-139 may inhibit PCa cell proliferation.

miR-139 induces $G_{0 / 1}$-phase cell cycle arrest in PCa cells. The three aforementioned PCa cell lines were transfected 

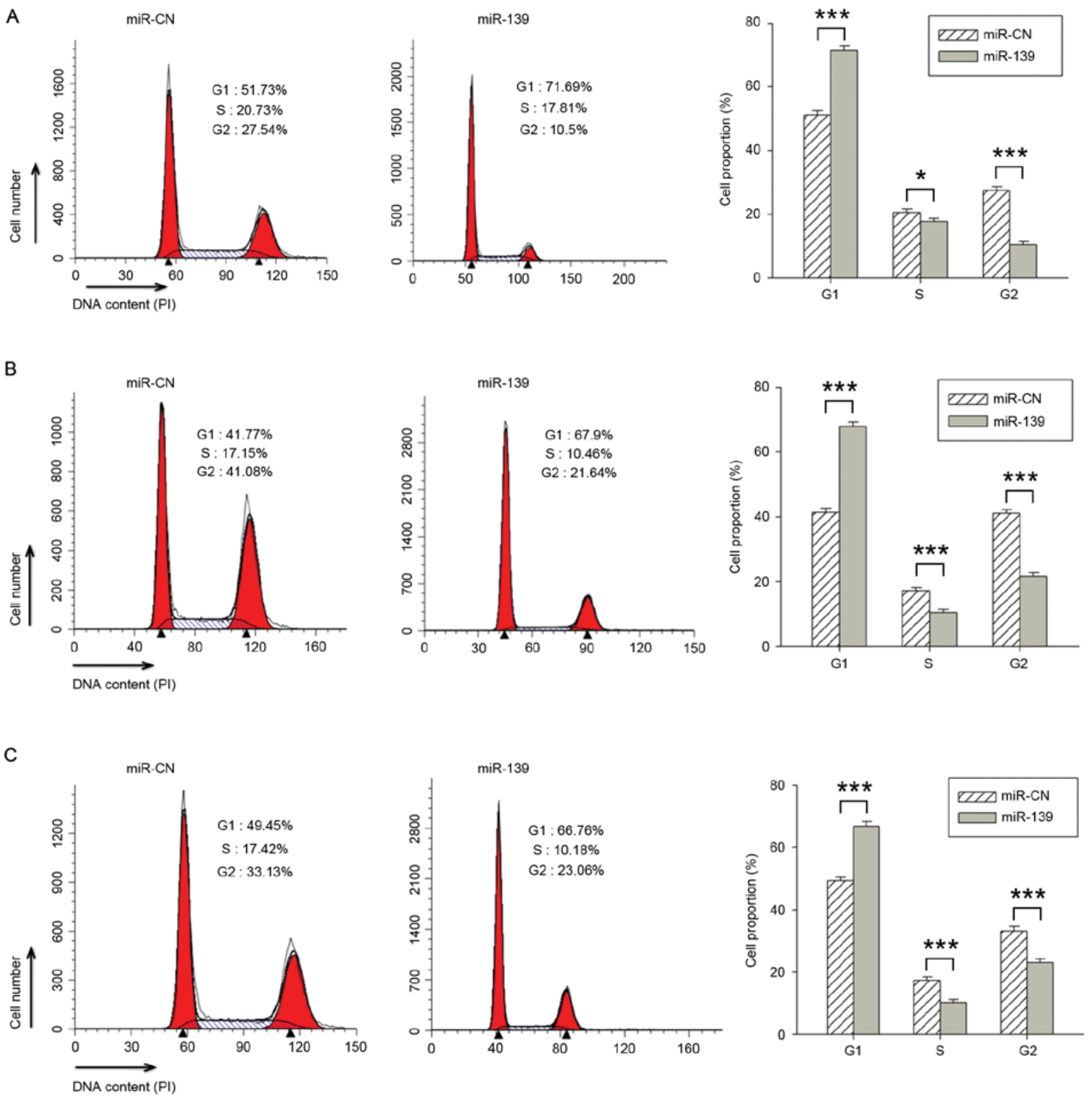

Figure 2. Cell cycle distribution of prostate cancer cells transfected with miR-CN or miR-139, assessed by flow cytometry. (A) PC-3 cells. (B) C4-2B cells. (C) LNCaP cells. "P $<0.05,{ }^{* * *} \mathrm{P}<0.001$ vs. miR-CN. miR, microRNA; CN, negative control; PI, propidium iodide.

with miR-139 or the miR-CN, and the cell cycle distribution was assessed using flow cytometry. The distribution results differed between transfected and control cells. The proportion of miR-139 transfected cells in $\mathrm{G}_{1}, \mathrm{~S}$ and $\mathrm{G}_{2}$ phase were $71.38 \pm 1.52,17.63 \pm 1.04$ and $10.43 \pm 0.96 \%$, respectively, in $\mathrm{PC}-3$ cells (Fig. 2A); 67.86 $\pm 1.46,10.45 \pm 0.98$ and $21.62 \pm 1.08 \%$, respectively, in $\mathrm{C} 4-2 \mathrm{~B}$ cells (Fig. $2 \mathrm{~B}$ ); and $66.74 \pm 1.52$, $10.18 \pm 0.93$ and $23.04 \pm 1.08 \%$ in LNCaP cells (Fig. 2C), respectively. The cells transfected with miR-139 exhibited a significantly increased percentage of cells in the $\mathrm{G}_{1}$ phase, and a decreased percentage in the $S$ and $G_{2}$ phases, compared with cells transfected with miR-CN $(\mathrm{P}<0.001)$. It was therefore concluded that miR-139 inhibited the proliferation of PCa cells by inducing $\mathrm{G}_{0 / 1}$-phase cell cycle arrest.

Effect of miR-139 on PCa cell apoptosis. The early apoptosis rates as determined by flow cytometry at $48 \mathrm{~h}$ after the transfection of the three PCa cell lines with miR-139 were $5.48 \%$ in PC-3 cells, $4.76 \%$ in C4-2B cells and $6.40 \%$ in LNCaP cells (data not shown). Compared with the control groups, the early apoptosis rates exhibited no significant difference. Therefore, miR-139 may have no effect on PCa cell apoptosis.

miR-139 reduces cell migration and invasiveness. The three PCa cell lines were transfected with miR-139 or the miR-CN, and the degree of cell migration was measured after $48 \mathrm{~h}$. Wound healing was only observed in the miR-CN group. The mean migration speeds of the negative control groups were $322.47 \pm 5.01,181.33 \pm 8.79$ and $506.72 \pm 4.22 \mu \mathrm{m} / \mathrm{min}$ in the PC-3, C4-2B and LNCaP cell lines, respectively; those of the miR-139-transfected cells were $100.5 \pm 4.77,102.2 \pm 2.86$ and $201.2 \pm 4.58 \mu \mathrm{m} / \mathrm{min}$ in PC-3, C4-2B and LNCaP cell lines, respectively. The migration speed was significantly lower in the groups transfected with miR-139 compared with the 


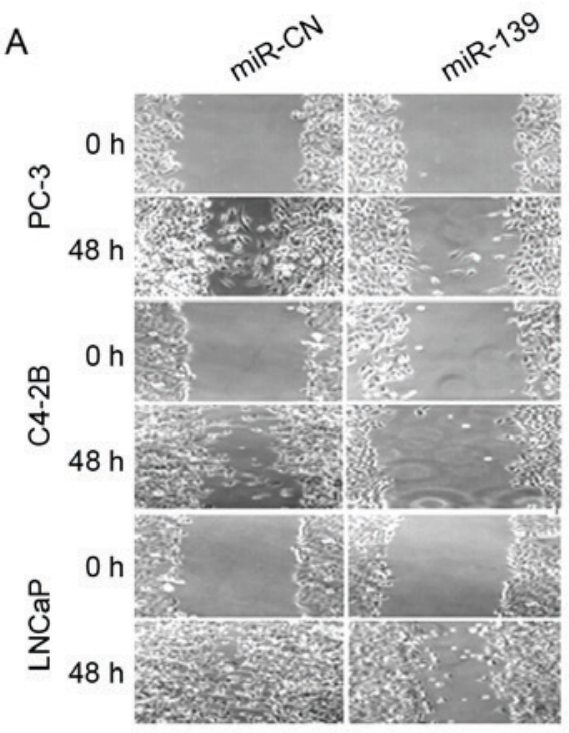

B

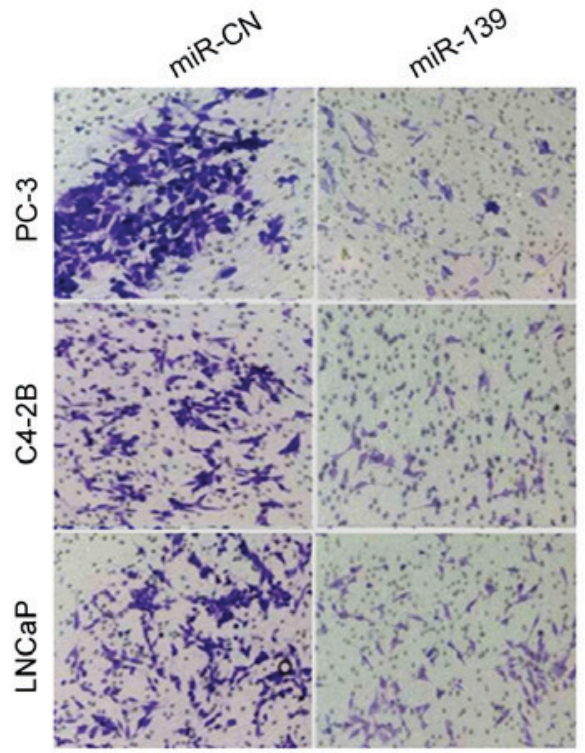

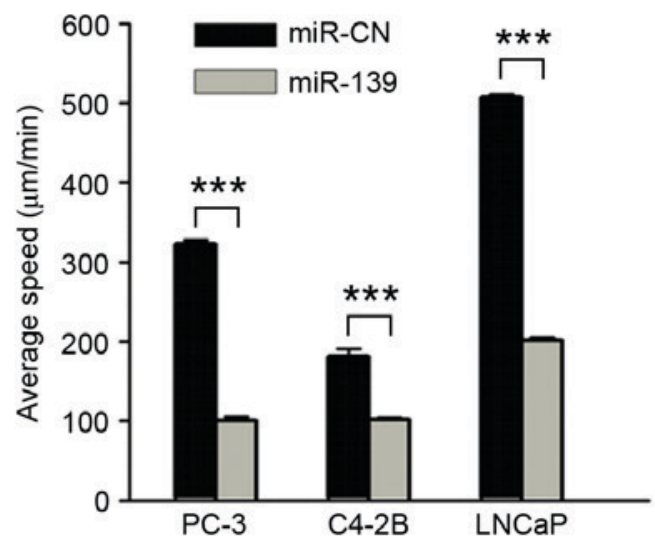

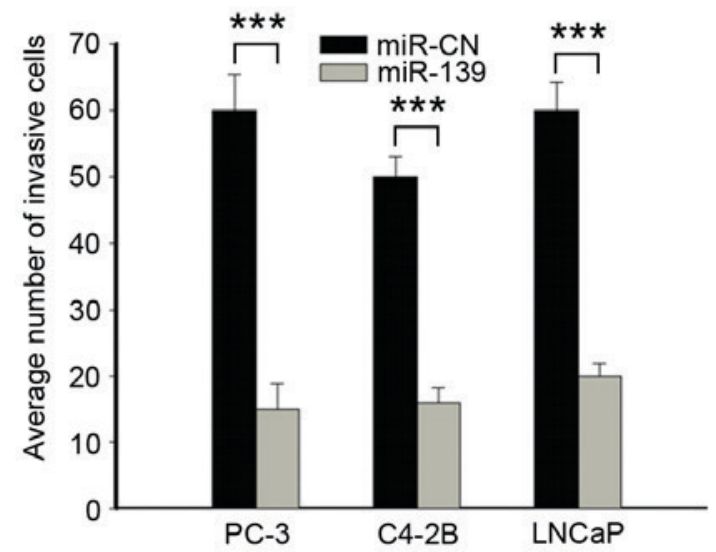

Figure 3. Cell migration and invasiveness of prostate cancer cells transfected with miR-CN or miR-139. (A) Light microscope image of cell migration and mean cell migration speed distribution in PC-3, C4-2B and LNCaP cells. Representative images are shown at x100 magnification. (B) Light microscope image of cell invasiveness and mean number of invasive cells per field in PC-3, C4-2B and LNCaP cells. Representative images are shown at 100x magnification. ${ }^{* * * *} \mathrm{P}<0.001$ vs. miR-CN. miR, microRNA; CN, negative control.

control groups $(\mathrm{P}<0.001$; Fig. 3A). Similarly, cell invasiveness for each cell line following transfection with miR-139 or the negative control was also observed using a Matrigel transwell assay. The results revealed a significant difference between the miR-139 and miR-NC groups ( $\mathrm{P}<0.001$; Fig. 3B). Consequently, it was concluded that cell migration and invasion were reduced by transfection with miR-139.

miR-139 decreases the expression of the target genes Notch1 and cyclin D1. Complementary base pairing was found between the Notch1 3'UTR and miR-139, as discovered by computational analysis (Fig. 4A), and a dual luciferase reporter assay revealed that luciferase activity was significantly lower in C4-2B and PC-3 cells when co-transfected with a Notch1 wild-type 3'UTR and miR-139 compared with transfection with a mutant Notch1
3'UTR and miR-139 ( $\mathrm{P}<0.001$; Fig. 4B and C). These results indicated that miR-139 was bound to the Notch1 3'UTR sequence. In addition, western blot analysis revealed that the levels of Notch1, matrix metalloproteinase (MMP)7, MMP9 and cyclin D1 in miR-139-transfected cells were decreased compared with those in the negative control group (Fig. 4D and E). Ultrastructural observation by transmission electron microscopy revealed the degranulation of the rough endoplasmic reticulum (ER) and mitochondrial swelling in PC-3 and C4-2B cells transfected with miR-139 (Fig. 5A and B).

\section{Discussion}

The majority of early-stage PCatumors are hormone-dependent, so androgen deprivation therapy is the conventional treatment. 
A

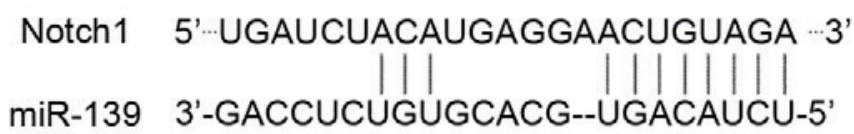

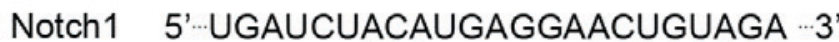

miR-139 3'-GACCUCUGUGCACG--UGACAUCU-5'

\section{B $\quad$ Control 3' UTR Notch1 3' UTR}
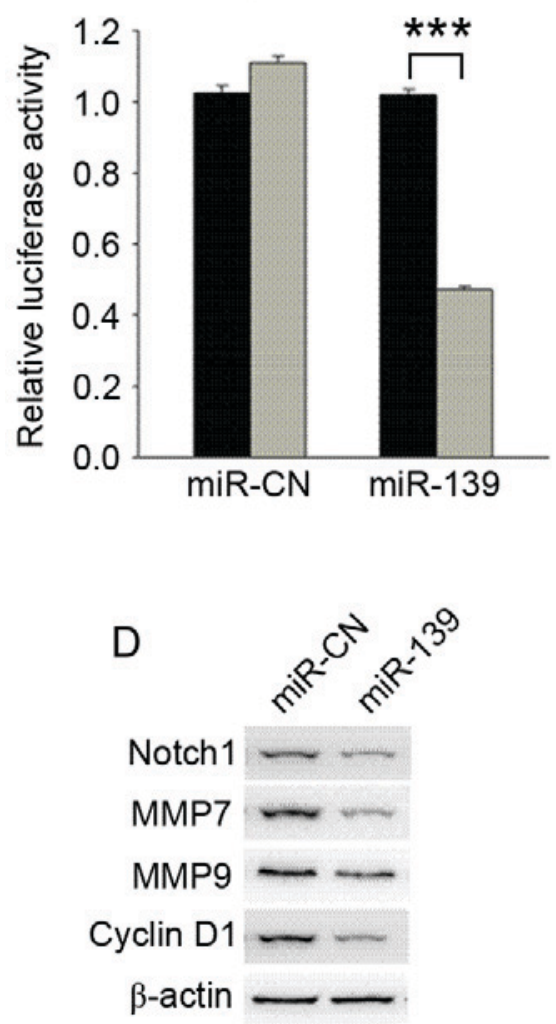
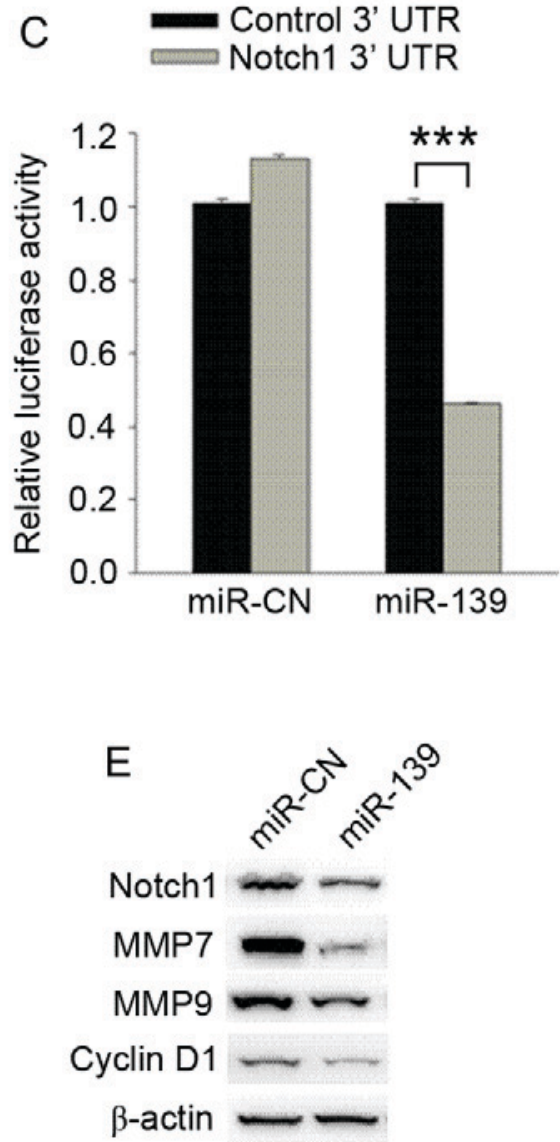

Figure 4. Notch1 is a direct target of miR-139 in prostate cancer cells. (A) Complementary base pairing between the Notch1 3'UTR and miR-139, as discovered by computational analysis. Relative luciferase activity in (B) PC-3 cells and (C) C4-2B cells. following co-transfection with either miR-139 and wild-type Notch1, or miR-139 and Notch1 with a mutant 3'UTR. Decrease in Notch1, MMP7, MMP9 and cyclin D1 in (D) PC-3 and (E) C4-2B cells by transfection with miR-139, as assessed using western blot analysis. ${ }^{* * * *} \mathrm{P}<0.001$ vs. mutant 3'UTR. miR, microRNA; CN, negative control; 3'UTR, 3'-untranslated region; MMP, matrix metalloproteinase.

A

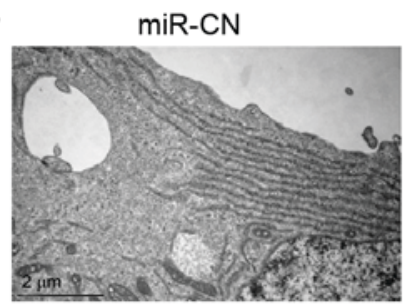

B

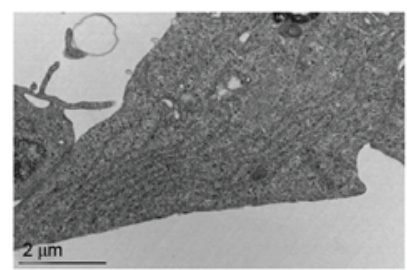

miR-139

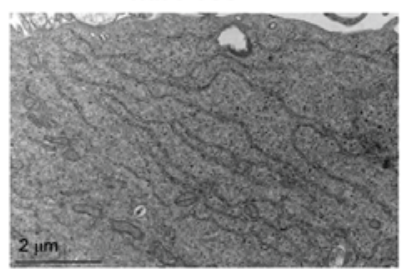

$\operatorname{miR}-139$

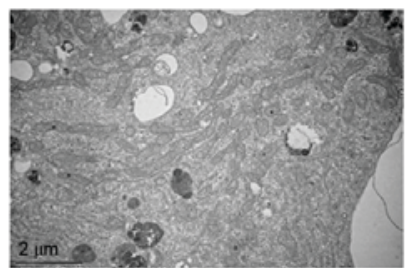

Figure 5. Ultrastructural observation revealed degranulation of rough endoplasmic reticulum and mitochondrial swelling in miR-139-transfected cells. Transmission electron micrographs of (A) PC-3 cells and (B) C4-2B cells. miR, microRNA; CN, negative control.

However, $>50 \%$ of patients will experience recurrence and metastasis or develop androgen-independent disease. The failure of endocrine therapy or the development of chemotherapy resistance leads to a reduction in quality of life and a decrease in survival time for the affected patients (3). A number of previous studies have demonstrated that miRNA dysregulation is a common event in tumor tissues. miRNAs are highly conserved, non-protein-coding, small, single-stranded RNAs that widely occur in eukaryotic genomes. Genetic variants in miRNA genes may alter miRNA expression and function, affecting human disease $(12,13)$. Previous studies have revealed that miRNAs participate in a number of physiological and pathological processes by degrading target mRNAs. For example, in tumorigenesis and cancer development, different miRNAs can either promote or inhibit the expression of oncogenic genes $(5,14)$. In addition to functioning as biomarkers, miRNAs may become direct targets for cancer treatments, potentially providing a novel direction for malignant tumor diagnosis, treatment and prognosis. Previous miRNA microarray analyses have revealed that miR-139 expression 
is downregulated in gastric cancer (15), colon cancer (16), breast cancer (17), liver cancer (18) and glioma (19), evidence of the association between reduced miR-139 expression and tumorigenesis; however, the expression of miR-139-5p in PCa remains unclear. Therefore, the present study aimed to investigate expression and biological functions of miR-139 in prostate cancer cells.

In the present study, the analysis of the cell cycle of cells transfected with miR-139 revealed a significantly increased percentage of cells in the $\mathrm{G}_{1}$ phase and a decreased percentage of cells in the $S$ and $G_{2}$ phases. miR-139 appeared to inhibit the proliferation of PCa cells by inducing $\mathrm{G}_{0 / 1}$-phase cell cycle arrest. Cell growth experiments in vitro confirmed that miR-139 significantly inhibited PCa cell proliferation. A previous study also revealed that miR-139 inhibited cell proliferation and induction of $\mathrm{G}_{0} /{ }_{1}$ arrest in colorectal cancer (9). Previous studies suggested that the expression of miR-139 induced apoptosis in colorectal cancer and glioma cells $(9,18)$. By contrast, the results of the present study demonstrated that miR-139 had no effect on apoptosis in all three PCa cell lines tested.

Mechanistic investigations by Zhang et al (9) and Song et al (20) revealed that miR-139 suppresses colorectal cancer proliferation by targeting Notch1 mRNA. Another previous study revealed that Notch signaling serves complicated functions in PCa (21). The luciferase reporter assay conducted in the present study revealed that luciferase activity was significantly decreased in C4-2B and PC-3 cells co-transfected with the wild-type Notch1 3'UTR and miR-139. The results indicated that miR-139 bound Notch1 directly in PCa cells. In addition, western blot analysis revealed that the levels of Notch1 and cyclin D1 protein in miR-139 transfected cells were markedly lower. As cyclin D1 has been demonstrated as a direct target of Notch1 in breast cancer (22), we hypothesized that miR-139 also targeted Notch1 and regulated the expression of cyclin D1 in PCa. MMP7 and MMP9 are involved in wound healing and tumor malignancy $(23,24)$, so the decreased levels of MMP7 and MMP9 in miR-139-transfected cells supported the conclusion that transfection with miR-139 reduced cell migration and malignancy.

A previous study suggested that the mitochondria-associated ER membrane functions as a platform for various intracellular stress responses, including apoptotic signaling, inflammatory signaling, the autophagic response and the unfolded protein response, and dysregulation of these signaling pathways may be associated with cancer cell metabolism (25). ER-associated protein degradation may act as a key regulatory factor that decides cell fate in breast cancer (26). The present study observed rough ER degranulation and mitochondrial swelling in miR-139-transfected PCa cells, although the mechanism by which this occurred is unknown. The ultrastructural changes may be associated with protein interactions between Notch1 and cyclin D1.

Evidence suggests that other miRNAs also serve an important function in PCa. Cohort research has suggested that it is possible to use the measurement of 14 miRNAs as a combined 'miR Score' to identify low-risk aggressive PCa (27). For instance, the decreased expression of miRNA-128 in the serum and $\mathrm{PCa}$ tissue may be associated with the malignant progression of tumors and a decreased recurrence-free survival rate (28). miRNA-195 suppresses tumor cell proliferation and metastasis by directly modulating the expression of breast cancer-overexpressed gene 1 (29), while suppressing cell migration and invasion by targeting FOS-like 1 expression in PCa (30). By contrast, miRNA-556-5p functions as an onco-miRNA and promotes prostate cancer cell growth by suppressing protein phosphatase 2 regulatory subunit B- $\alpha$ (PPP2R2A) expression. Previous experimental data have demonstrated that the ectopic expression of miRNA-556-5p results in the downregulation of PPP2R2A protein, which in turn results in the downregulation of cyclin dependent kinase inhibitor $1 \mathrm{~B}$ and the upregulation of cyclin D1 (31). The molecular interaction networks between different miRNAs, their respective target proteins and the complete cancer-associated mechanisms underlying the effect of miRNAs remain to be clarified.

In summary, to the best of our knowledge, the present study revealed for the first time that miR-139 reduces cyclin D1 expression and inhibits cell proliferation through targeting Notch1 in PCa. Furthermore, MMP7 and MMP9 expression was downregulated in miR-139-transfected PCa cells. These data suggested that this pathway may be a potential therapeutic target for PCa treatment.

\section{Acknowledgements}

The authors would like to thank Dr Qi-Lin Ao (Tongji Medical College, Huazhong University of Science and Technology, Wuhan, China) for reviewing histology data.

\section{Funding}

The present study was supported by the National Science Foundation of China (grant nos. 81772775, 81472783, 81630060, 81572571 and 81372801).

\section{Availability of data and materials}

The datasets used and/or analyzed during the current study are available from the corresponding author on reasonable request.

\section{Authors' contributions}

QS and JW conceived and designed the study. QS, DW, DL, PW, GC performed the literature search, data extraction and statistical analysis and drafted the paper. KL, SL, XB, CF performed the experiments. JW supervised the literature search, data extraction and analysis, and reviewed the paper. All authors have read and approved the final manuscript.

\section{Ethics approval and consent to participate}

Tissue samples were obtained and handled in accordance with a protocol approved by the Institutional Review Board for Human Research of Tongji Hospital (Wuhan, China). Written informed consent was obtained from all patients.

\section{Consent for publication}

Not applicable.

\section{Competing interests}

The authors declare that they have no competing interests. 


\section{References}

1. Siegel RL, Miller KD and Jemal A: Cancer statistics, 2015. CA Cancer J Clin 65: 5-29, 2015.

2. Chen W, Zheng R, Zeng H, Zhang S and He J: Annual report on status of cancer in China, 2011. Chin J Cancer Res 27: 2-12, 2015.

3. Wei J, Wang Z, Makarov D and Li X: Current treatments and novel therapeutic targets for castration resistant prostate cancer with bone metastasis. Am J Clin Exp Urol 1: 30-38, 2013.

4. Kelly BD, Miller N, Sweeney KJ, Durkan GC, Rogers E, Walsh K and Kerin MJ: A circulating MicroRNA signature as a biomarker for prostate cancer in a high risk group. J Clin Med 4: 1369-1379, 2015.

5. Pillai RS: MicroRNA function: Multiple mechanisms for a tiny RNA? RNA 11: 1753-1761, 2005

6. Carthew RW and Sontheimer EJ: Origins and mechanisms of miRNAs and siRNAs. Cell 136: 642-655, 2009.

7. Zhang HD, Jiang LH, Sun DW, Li J and Tang JH: MiR-139-5p: Promising biomarker for cancer. Tumour Biol 36: 1355-1365, 2015.

8. Xu W, Hang M, Yuan CY, Wu FL, Chen SB and Xue K: MicroRNA-139-5p inhibits cell proliferation and invasion by targeting insulin-like growth factor 1 receptor in human non-small cell lung cancer. Int J Clin Exp Pathol 8: 3864-3870, 2015.

9. Zhang L, Dong Y, Zhu N, Tsoi H, Zhao Z, Wu CW, Wang K, Zheng S, Ng SS, Chan FK, et al: microRNA-139-5p exerts tumor suppressor function by targeting NOTCH1 in colorectal cancer. Mol Cancer 13: 124, 2014.

10. Zhang HD, Sun DW, Mao L, Zhang J, Jiang LH, Li J, Wu Y, Ji H, Chen W, Wang J, et al: MiR-139-5p inhibits the biological function of breast cancer cells by targeting Notch1 and mediates chemosensitivity to docetaxel. Biochem Biophys Res Commun 465: 702-713, 2015

11. Livak KJ and Schmittgen TD: Analysis of relative gene expression data using real-time quantitative PCR and the 2(-Delta Delta C(T)) method. Methods 25: 402-408, 2001

12. Cammaerts S, Strazisar M, De Rijk P and Del Favero J: Genetic variants in microRNA genes: Impact on microRNA expression, function, and disease. Front Genet 6: 186, 2015.

13. Zhou Y, Du WD, Chen G, Ruan J, Xu S, Zhou FS, Zuo XB, Lv ZJ and Zhang XJ: Association analysis of genetic variants in microRNA networks and gastric cancer risk in a Chinese Han population. J Cancer Res Clin Oncol 138: 939-945, 2012.

14. Chen B, Li H, Zeng X, Yang P, Liu X, Zhao X and Liang S: Roles of microRNA on cancer cell metabolism. J Transl Med 10: 228, 2012.

15. Bao W, Fu HJ, Xie QS, Wang L, Zhang R, Guo ZY, Zhao J, Meng YL, Ren XL, Wang T, et al: HER2 interacts with CD44 to up-regulate CXCR4 via epigenetic silencing of microRNA-139 in gastric cancer cells. Gastroenterology 141: 2076-2087.e6, 2011.

16. Shen K, Mao R, Ma L, Li Y, Qiu Y, Cui D, Le V, Yin P, Ni L and Liu J: Post-transcriptional regulation of the tumor suppressor miR-139-5p and a network of miR-139-5p-mediated mRNA interactions in colorectal cancer. FEBS J 281: 3609-3624, 2014.

17. Krishnan K, Steptoe AL, Martin HC, Pattabiraman DR, Nones K, Waddell N, Mariasegaram M, Simpson PT, Lakhani SR, Vlassov A, et al: miR-139-5p is a regulator of metastatic pathways in breast cancer. RNA 19: 1767-1780, 2013.
18. Li T, Yin J, Yuan L, Wang S, Yang L, Du X and Lu J: Downregulation of microRNA-139 is associated with hepatocellular carcinoma risk and short-term survival. Oncol Rep 31: 1699-1706, 2014

19. Li RY, Chen LC, Zhang HY, Du WZ, Feng Y, Wang HB, Wen JQ, Liu X, Li XF, Sun Y, et al: MiR-139 inhibits Mcl-1 expression and potentiates TMZ-induced apoptosis in glioma. CNS Neurosci Ther 19: 477-483, 2013

20. Song M, Yin Y, Zhang J, Zhang B, Bian Z, Quan C, Zhou L, Hu Y, Wang Q, Ni S, et al: MiR-139-5p inhibits migration and invasion of colorectal cancer by downregulating AMFR and NOTCH1. Protein Cell 5: 851-861, 2014.

21. Deng G, Ma L, Meng Q, Ju X, Jiang K, Jiang P and Yu Z: Notch signaling in the prostate: Critical roles during development and in the hallmarks of prostate cancer biology. J Cancer Res Clin Oncol 142: 531-547, 2016.

22. Cohen B, Shimizu M, Izrailit J, Ng NF, Buchman Y, Pan JG, Dering $\mathrm{J}$ and Reedijk M: Cyclin D1 is a direct target of JAG1-mediated Notch signaling in breast cancer. Breast Cancer Res Treat 123: 113-124, 2010.

23. Hsu TI, Lin SC, Lu PS, Chang WC, Hung CY, Yeh YM, Su WC, Liao PC and Hung JJ: MMP7-mediated cleavage of nucleolin at Asp255 induces MMP9 expression to promote tumor malignancy. Oncogene 34: 826-837, 2015

24. Wong VW, Garg RK, Sorkin M, Rustad KC, Akaishi S, Levi K, Nelson ER, Tran M, Rennert R, Liu W, et al: Loss of keratinocyte focal adhesion kinase stimulates dermal proteolysis through upregulation of MMP9 in wound healing. Ann Surg 260: 1138-1146, 2014

25. Kato $\mathrm{H}$ and Nishitoh H: Stress responses from the endoplasmic reticulum in cancer. Front Oncol 5: 93, 2015.

26. Fan P, Cunliffe HE, Maximov PY, Agboke FA, McDaniel RE, Zou X, Ramos P, Russell ML and Jordan VC: Integration of downstream signals of insulin-like growth factor-1 receptor by endoplasmic reticulum stress for estrogen-induced growth or apoptosis in breast cancer cells. Mol Cancer Res 13: 1367-1376, 2015.

27. Mihelich BL, Maranville JC, Nolley R, Peehl DM and Nonn L: Elevated serum microRNA levels associate with absence of high-grade prostate cancer in a retrospective cohort. PLoS One 10: e0124245, 2015.

28. Sun X, Yang Z, Zhang Y, He J, Wang F, Su P, Han J, Song Z and Fei Y: Prognostic implications of tissue and serum levels of microRNA-128 in human prostate cancer. Int J Clin Exp Pathol 8: 8394-8401, 2015.

29. Guo J, Wang M and Liu X: MicroRNA-195 suppresses tumor cell proliferation and metastasis by directly targeting BCOX1 in prostate carcinoma. J Exp Clin Cancer Res 34: 91, 2015.

30. Wu J, Ji A, Wang X, Zhu Y, Yu Y, Lin Y, Liu Y, Li S, Liang Z, Xu X, et al: MicroRNA-195-5p, a new regulator of Fra-1, suppresses the migration and invasion of prostate cancer cells. J Transl Med 13: 289, 2015

31. Zhao W, Cao L, Zeng S, Qin H and Men T: Upregulation of miR-556-5p promoted prostate cancer cell proliferation by suppressing PPP2R2A expression. Biomed Pharmacother 75: 142-147, 2015.

This work is licensed under a Creative Commons Attribution-NonCommercial-NoDerivatives 4.0 International (CC BY-NC-ND 4.0) License. 